\title{
Offensive or defensive play in soccer: a game-theoretical approach
}

\author{
Daniele Gambarelli ${ }^{1}$, Gianfranco Gambarelli ${ }^{2}$ and Dries Goossens ${ }^{3}$ \\ ${ }^{1}$ Five Schools Beach Volley Associazione, Sportiva Dilettantistica, Cesenatico (FC), Emilia \\ Romagna, Italy, e-mail: daniele.gambarelli@gmail.com \\ ${ }^{2}$ Gianfranco Gambarelli: Università degli Studi di Bergamo, Department of Management, \\ Economics and Quantitative Methods, Bergamo, Lombardia, Italy, e-mail: \\ gianfranco.gambarelli@unibg.it
}

${ }^{3}$ Ghent University, Department of Business Informatics and Operations Management, Gent, Belgium, e-mail: dries.goossens@ ugent.be

Important note: This is an author-version of the article published in Journal of Quantitative Analysis in Sports (De Gruyter). To refer to or to cite this work, please use the citation to the published version (see https://doi.org/10.1515/jqas-2017-0071).

\begin{abstract}
In many sports, such as soccer, a coach has to decide whether to adopt an "offensive" or a "defensive" approach to a match. This strategic decision depends on the strengths and weaknesses of his team with respect to the opponent, but also on the strategy chosen by the opponent's coach. The fact that a draw also results in a point, which may be important for the ranking, further complicates this decision, as the coach must assess whether it is better to adopt a defensive team formation in order to obtain a draw, or to aim for victory despite the increased risk of defeat.

We study this topic by means of a game-theoretical approach, and we develop an algorithm which allows to compute the optimal strategy. The algorithm tests the stability of the outcome with respect to uncertainty in the estimated win probabilities. Furthermore, our approach can handle in-game events, which may provoke changes in optimal strategy. Finally, our model also allows us to assess the effect of various point systems on the willingness of teams to opt for attacking play. Our method is applied to a case study, based on the match Italy - Costa Rica (World Cup 2014).
\end{abstract}

Key words: Game Theory, Soccer, Strategy, Rule Change, Team Formation.

\section{Introduction}

A soccer match ends after 90 minutes with either a winning and a losing team, or undecided (i.e. a draw or a tie). Before the start of the match, a coach needs to decide on the team's strategy as well as the team formation most suited to implement that strategy. Although there is the possibility to adjust the strategy during the match, the initial choice often affects the outcome of the match. Several tactical variants are conceivable, however, in general we distinguish an offensive and a defensive strategy. Informally, an offensive strategy can be described as more focused on scoring goals, whereas a defensive play emphasizes avoiding conceded goals. A coach will determine the 
choice of strategy based on the strengths and weaknesses of his teams, and those of the opponent. The fact that one team's strategy affects the decision of the other team, is an important complication for the coach. Hence, a game theoretic approach can be useful for tackling this type of problem.

In a round robin tournament, where each team plays against each other team the same number of times, points are awarded for a win, a draw, and a loss. The ranking of each team is then based on the sum of points it collected in all its matches. Since scoring a goal is a necessary condition to win a game, and not conceding a goal is sufficient for a draw, there is a link between the choice of strategy and the value that a draw has for the ranking. Apart from special circumstances, where a draw may be sufficient to qualify (and a win does not offer any added value), the importance of a draw is determined by the number of points it brings, relative to the number of points for a win. For a long time, the 2-point standard (a.k.a. the 2-1-0 award system) was used, which awards two points to the winning team, no points to the losing team, and in case of a draw, each team receives one point. In 1981, England became the first country to award the winning team with 3 points (i.e. the 3-1-0 award system). The motivation for this rule change, which stimulates teams to win a match rather than being satisfied with a draw, was to encourage attacking play, which is typically experienced as more attractive by the fans. It was not until after the use of this rule in the 1994 FIFA World Cup, that countries like France and Italy also implemented the 3-1-0 point score system. In 1995, FIFA formally adopted the system and it subsequently became standard in international tournaments, as well as in most domestic leagues.

In this paper, we study the decision for offensive or defensive play by means of a game-theoretical approach. After a short overview of related literature (Section 2), we develop a model that, given estimates on the win probabilities for each combination of strategies adopted by the opponents, results in a strategic advice for each team (Section 3). This model also allows us to observe the impact of various point systems on the willingness of teams to play offensively. Section 4 provides an algorithm for the automatic computation of the optimal strategy, which takes into account uncertainty about the win probability estimates. We apply our method to the 2014 World Cup match Italy - Costa Rica in Section 5, before coming to the conclusions.

\section{Literature review}

In a game, players take actions according to the rewards they expect to gain from their behavior. Game theory is the science of optimal decision-making of independent and competing players in a strategic setting. For those wishing to find out more about game theory and relative historical grounds, reference should be made to Owen (2013) and Gambarelli and Owen (2004). Although game theory is deeply rooted in economic issues, there are several applications to sports strategy, including e.g. play calling in American Football (Jordan et al., 2009) pitting in NASCAR racing (Deck et al., 2014), or first serves in tennis (Walker and Wooders, 2001).

Game theory also received a fair share of attention in soccer (see for instance the entertaining book by Palacios-Huerta (2014)). Hiller (2015) applied the approach of Hernández-Lamoneda and Sánchez-Sánchez (2010) which is based on cooperative game theory to soccer, creating a player performance metric which captures the contribution of the player to the success of his team. Auer and Hillier (2015) computed this measure for the German Bundesliga teams in the season 2012-13, and found that it correlates positively with classic player performance measures such as e.g. overall player scores published by prominent sports magazines. 
A penalty kick can also be studied from a game theory perspective, when seen as a two-person zero-sum game (the goalkeeper loses whilst the penalty taker wins, or vice versa). Using data from penalty kicking in the Bundesliga, Dohmen and Sonnabend (2018) provide evidence that experienced players in high-stakes environments behave according to game theoretic predictions. They verify empirically that the main requirements for the existence of a Nash equilibrium in mixed strategies are met (i.e. the expected payoffs are equal across strategies and players choose their actions randomly). A game theoretic analysis of penalty kicks was also performed by Chiappori et al. (2002), Coloma (2007), and Palacios-Huerta (2003).

Game theory has also been applied to rule design in soccer. For instance, Brams and Ismail (2016) show that the current rule for penalty shootouts, where a coin toss determines which team kicks first on all five penalty kicks, gives a substantial advantage to the first-kicking team, both in theory and in practice. They present the so-called catch-up rule to mitigate this bias.

When we focus on the use of game theory for strategical/tactical decisions to be taken by soccer coaches of two opposing teams, including the effect of interaction between their decisions, the literature includes just a few contributions.

Hirotsu and Wright (2006) study the interaction between both teams' tactical changes before and during the game, which boil down to a change of formation imposed by the manager. They see the selection of a formation as one of the main tactics employed by managers to make their team play offensively or defensively. The authors model a soccer match as two-player zero-sum game, where one player's loss is the other's gain, in order to quantify the effect of decisions to change the team formation on the probability of winning the match. They demonstrated that such tactical decisions can indeed influence the probability of winning the game, using real data from the Japanese professional soccer league, and illustrating it on a number of match cases. The results show that the difference between the combinations of the best and worst tactics leads to a significant difference in terms of the probability of winning.

Hirotsu et al. (2009) extend the model introduced by Hirotsu and Wright (2006) to non-zero sum games, this time focusing on the expected number of points gained in a match. A non-zero sum game is natural given the 3-1-0 scoring rule, which distributes three points for a match with a winner (all points go to the winner), whereas a draw only yields two points shared among the teams. They demonstrate the effect of the point score system on tactical changes, as well as the effect of cooperation between managers which could make both teams benefit in terms of the expected number of league points gained in a match. Note that neither Hirotsu and Wright (2006), nor Hirotsu et al. (2009) deal with the case where the game is solved using mixed strategies, in which teams select their tactics according to probabilities.

The point system, and in particular the switch from the 2-1-0 to the 3-1-0 system, has been the topic of several studies, theoretical as well as empirical. Using a game theoretic approach, Brocas and Carillio (2004) find that the 3-1-0 rule results in more defensive play in the first half and more attacking play in the last part of a match, as opposed to the 2-1-0 rule where the teams do not change their strategy. Haugen (2008) proves that, under some reasonable assumptions, in a match between two equal teams, no team will choose to alter its strategy in a more defensive direction after the switch to the 3-1-0 system. Moreover, certain parameter value (team differences) will result in more offensive play. 
While the theoretical work on the scoring rule points more or less in the same direction, empirical studies offer more diverse results. Fernandez-Cantelli and Meeden (2002) performed an empirical study involving soccer leagues in 10 countries. They find little evidence to support that the switch to the 3-1-0 system led to more goals or fewer ties; they report a noteworthy decrease in ties only for Italy and Turkey. Dilger and Geyer (2009) do find evidence for a decrease in matches ending in a tie after the rule change for the German Bundesliga. This difference is significant, even when compared with matches from the German Cup (being a knock-out tournament, the German Cup was not influenced by the switch to the 3-point system). Then again, Aylott and Aylott (2007) show that the average number of goals per match (with the exception of the German Bundesliga), as well as the number of draws increased, since the introduction of the 3-1-0 rule. Dewenter and Emami Namini (2013) argue that when the reward for a win is increased, the opportunity costs of playing extremely offensively rise, and consequently, the home team chooses a more defensive playing style. Hence, an increase in the rewards for a win leads to the counterintuitive result of more defensive play if the home bias is sufficiently strong and if the levels of offensiveness of the teams are strategic substitutes. The authors find evidence for their theoretical hypotheses in the German Bundesliga: the 3-1-0 rule leads to a reduction in both the number of goals and the number of victories for home teams. The away teams, in contrast, choose a more offensive strategy (the total number of goals as well as the total number of victories have not been affected significantly).

\section{A game-theoretical approach}

In this section, we develop a game-theoretical model that, given estimates on the win probabilities for each combination of strategies adopted by the opponents, results in an strategic advice for each opponent. We assume that each team has two discrete strategic choices: an offensive (O) or defensive (D) approach.

We will now describe the input parameters, labelling the two teams $A$ and $B$. We indicate $\alpha_{D D}$ and $\beta_{D D}$ respectively as the probabilities of a win for team $\mathrm{A}$ and team $\mathrm{B}$ if both adopt a defensive strategy $\left(\alpha_{D D}+\beta_{D D} \leq 1\right)$; the probability of a draw will, therefore, correspond to $1-\alpha_{D D}-\beta_{D D}$. In the same way, we define $\alpha_{O O}$ and $\beta_{O O}$ (both teams with an offensive approach), $\alpha_{D O}$ and $\beta_{D O}$ ( $A$ in defense and $B$ in attack), $\alpha_{O D}$ and $\beta_{O D}(A$ in attack and $B$ in defense).

We define the expected payoff $a_{i j}\left(b_{i j}\right)$ for team A (B) where team A follows strategy $i$ and team $\mathrm{B}$ adopts strategy $\mathrm{j}$ (with $i, j \in\{\mathrm{O}, \mathrm{D}\}$ ). The payoffs are calculated as follows, based on the hypothesis that a win awards $p$ points and a draw yields $q$ points:

$a_{i j}=p \alpha_{i j}+q\left(1-\alpha_{i j}-\beta_{i j}\right)=q+(p-q) \alpha_{i j}-q \beta_{i j}$

$b_{i j}=p \beta_{i j}+q\left(1-\alpha_{i j}-\beta_{i j}\right)=q+(p-q) \beta_{i j}-q \alpha_{i j}$

We are facing a normal-form variable-sum game. The competitive solution by John Nash (1950) requires the use of an algorithm of Linear Programming on the matrices $\left[\alpha_{i j}\right]$ and $\left[\beta_{i j}\right]$ based on John von Neumann's Minimax Theorem (1928). However, the specificity of the problem considered here allows closed solutions which we will present in this section for the reader's convenience. Firstly, it is necessary to verify whether any team has a dominant strategy (i.e. a strategy that results in a higher expected payoff than the other strategy, regardless of the opponent's strategy), in which case the solution can easily be found. 
If there is no domination, the solution consists of in probability distributions $\left(q_{D}^{A}, q_{O}^{A}\right)$ on the strategies of $A$ and $\left(q_{D}^{B}, q_{O}^{B}\right)$ on the strategies of $B$. Such assignments of probabilities to the offensive and defensive strategy correspond to mixed strategies, and can also be interpreted as different levels of adoption of both strategies.

Let's define $h=\left(a_{O D}-a_{O O}\right) /\left(a_{D O}+a_{O D}-a_{D D}-a_{O O}\right)$. The probability distribution for the strategy of team $A$ is then as follows:

$q_{D}^{A}=h$ if $0 \leq h \leq 1$, and

$q_{D}^{A}=\left\{\begin{array}{l}0 \text { if } \min \left(a_{D D}, a_{D O}\right)<\min \left(a_{O D}, a_{O O}\right), \\ \left.1 \text { if } \min \left(a_{D D}, a_{D O}\right)\right)>\min \left(a_{O D}, a_{O O}\right) \text { elsewhere, } \\ \text { any value between } 0 \text { and } 1, \text { otherwise. }\end{array}\right.$

In all cases, $q_{O}^{A}=1-q_{D}^{A}$.

Similarly, for team B, with $k=\left(b_{O D}-b_{O O}\right) /\left(b_{D O}+b_{O D}-b_{D D}-b_{O O}\right)$, the distribution is given by:

$q_{D}^{B} \quad=k$ if $0 \leq k \leq 1$, and

$q_{D}^{B}=\left\{\begin{array}{l}0 \text { if } \min \left(b_{D D}, b_{O D}\right)<\min \left(b_{O D}, b_{O O}\right) \\ 1 \text { if } \min \left(b_{D D}, b_{O D}\right)>\min \left(b_{O D}, b_{O O}\right) \text { elsewhere, } \\ \text { any value between } 0 \text { and } 1 \text {, otherwise. }\end{array}\right.$

In all cases, $q_{O}^{B}=1-q_{D}^{B}$.

The expected payoffs of the two teams are:

$p^{A}=q_{D}^{A} q_{D}^{B} a_{D D}+q_{D}^{A} q_{O}^{B} a_{D O}+q_{O}^{A} q_{D}^{B} a_{O D}+q_{O}^{A} q_{O}^{B} a_{O O}$

$p^{B}=q_{D}^{A} q_{D}^{B} b_{D D}+q_{D}^{A} q_{O}^{B} b_{D O}+q_{O}^{A} q_{D}^{B} b_{O D}+q_{O}^{A} q_{O}^{B} b_{O O}$

Consider for example a setting where the expected payoffs for team A and B are as presented in Table 1.

\begin{tabular}{|c|c|c|}
\hline & Team B Defensive & Team B Offensive \\
\hline Team A Defensive & $(1.0,2.5)$ & $(1.8,0.7)$ \\
\hline Team A Offensive & $(1.5,1.0)$ & $(1.1,1.2)$ \\
\hline
\end{tabular}

Table 1: The expected payoffs for teams A and B. Each cell has values $\left(a_{i j}, b_{i j}\right)$ with team A adopting strategy $i$ and team B adopting strategy $j$. 
This means that for team A, $h=(1.5-1.1) /(1.8+1.5-1.0-1.1)=1 / 3$. Hence $q_{D}^{A}=1 / 3$ and $q_{O}^{A}$ $=2 / 3$, or in other words, in the long run, team A should attack in $2 / 3$ of such cases and defend in $1 / 3$ of such cases. For team $\mathrm{B}, k=(0.7-1.2) /(1.0+0.7-2.5-1.2)=1 / 4$, such that team $\mathrm{B}$ should attack with a probability of $75 \%\left(q_{O}^{B}=3 / 4\right)$ and choose a defensive approach with a probability of $25 \%\left(q_{D}^{B}=1 / 4\right)$.

An important remark concerns the incompleteness of the information on which the table of win probability estimates (i.e. $\alpha_{i j}$ and $\beta_{i j}$ ) are based. In fact, every coach has at his disposal inside information regarding the psychophysical condition of his players, but may not have the same information about the opposing team. Consequently, his win probability estimates could differ from that of the opponent's coach. Except in the cases when the two tables of probabilities lead to different results in terms of dominance, such differences do not influence the strategy chosen by the coach. In fact, only in such situations can the table of the opponent's coach cause problems. The possibility to repeat the calculation given by the algorithm that we will present in the next section, helps to overcome this problem.

\section{Implementation}

In this section, we present an algorithm for the automatic calculation of strategy advice (i.e. the solution to the model presented in section 3), with a test of the robustness of this advice with respect to uncertainty involved in estimating the win probabilities for each combination of strategies.

The input of the algorithm consists of the four pairs of probabilities of a win of team A and team B: $\alpha_{i j}$ and $\beta_{i j}$, with $i, j \in\{\mathrm{O}, \mathrm{D}\}$. While reasonably accurate predictions for the outcome (win, draw, loss) of a match can be derived from the odds published by bookmakers, this is much less the case for $\alpha_{i j}$ and $\beta_{i j}$, as they require insight in the impact of the strategies on the match outcome. As this input is chosen on the basis of a subjective evaluation of the available data, it is characterized by uncertainty. Obviously, wrong initial considerations can lead to poor strategic advice, and forecasting is strongly based on a priori achievable assessment. In order to give an indication of the robustness of the resulting strategy advise, we vary the starting conditions in a suitable neighborhood (necessarily a discrete set of points). We use the mean of the results in the neighborhood as a tractable way to summarize the strategies in the neighborhood. By comparing this mean with the initial result for increasing an increase size of the neighborhood (corresponding to an increase degree of uncertainty), we can see how much the resulting strategy can change due to increasing variations of the initial estimate of the win probabilities.

The neighborhood is generated as follows. We specify a value $r$ indicating how far we deviate from the original estimates for $\alpha_{i j}$ and $\beta_{i j}$, For each pair of values $\alpha_{i j}$ and $\beta_{i j}$, for some choice of $i, j \in\{\mathrm{O}, \mathrm{D}\}$, we are interested in 9 discrete points, centered around the values $\alpha_{i j}$ and $\beta_{i j}$, given in Table 2. As there are 4 pairs of $\left(\alpha_{i j}, \beta_{i j}\right)$ given the values that $i$ and $j$ can take, this results in a neighborhood with $9^{\wedge} 4(=6561)$ points. The algorithm computes the solution for each of these points. A solution consists of the probability distribution of the strategies $\left(q_{D}^{A}, q_{O}^{A}, q_{D}^{B}, q_{O}^{B}\right)$ as well as the resulting payoffs $\left(p^{A}, p^{B}\right)$ for both teams, and is computed in the way described in section 3 . The algorithm aggregates the information obtained in the neighborhood by outputting the mean of the solution parameters over all points in that neighborhood. 


\begin{tabular}{|l|c|}
\hline 1 & $\left(\alpha_{i j}, \beta_{i j}\right)$ \\
\hline 2 & $\left(\alpha_{i j}-r, \beta_{i j}-r\right)$ \\
\hline 3 & $\left(\alpha_{i j}-r, \beta_{i j}\right)$ \\
\hline 4 & $\left(\alpha_{i j}-r, \beta_{i j}+r\right)$ \\
\hline 5 & $\left(\alpha_{i j}, \beta_{i j}-r\right)$ \\
\hline 6 & $\left(\alpha_{i j}, \beta_{i j}+r\right)$ \\
\hline 7 & $\left(\alpha_{i j}+r, \beta_{i j}-r\right)$ \\
\hline 8 & $\left(\alpha_{i j}+r, \beta_{i j}\right)$ \\
\hline 9 & $\left(\alpha_{i j}+r, \beta_{i j}+r\right)$ \\
\hline
\end{tabular}

Table 2: Neighborhood for a pair $\left(\alpha_{\mathrm{ij}}, \beta_{\mathrm{ij}}\right)$, for a given $i, j$ and $r$.

A representation of the algorithm in pseudo code is given in Figure 1. The algorithm uses the data structure storage to store all solutions obtained in a neighborhood determined by the value $r$, where $r$ takes on values from 0.01 to radius, which establishes the maximum radius of the neighborhood to be searched. This allows us to evaluate how the solution varies as the win probabilities $\alpha_{i j}$ and $\beta_{i j}$ move further away from our original estimate. Note that in case radius=0, only the solution for the given values of $\alpha_{i j}$ and $\beta_{i j}$ is calculated.

Note that this robustness analysis is not intended to account for future match events match (e.g. a goal scored or a red card). Indeed, when events occur during the match, the analysis should be redone based on updated values of $\alpha_{i j}$ and $\beta_{i j}$. Nevertheless, these new win probabilities will also be subject to uncertainty. Hence, redoing the robustness analysis after each such event is advised.

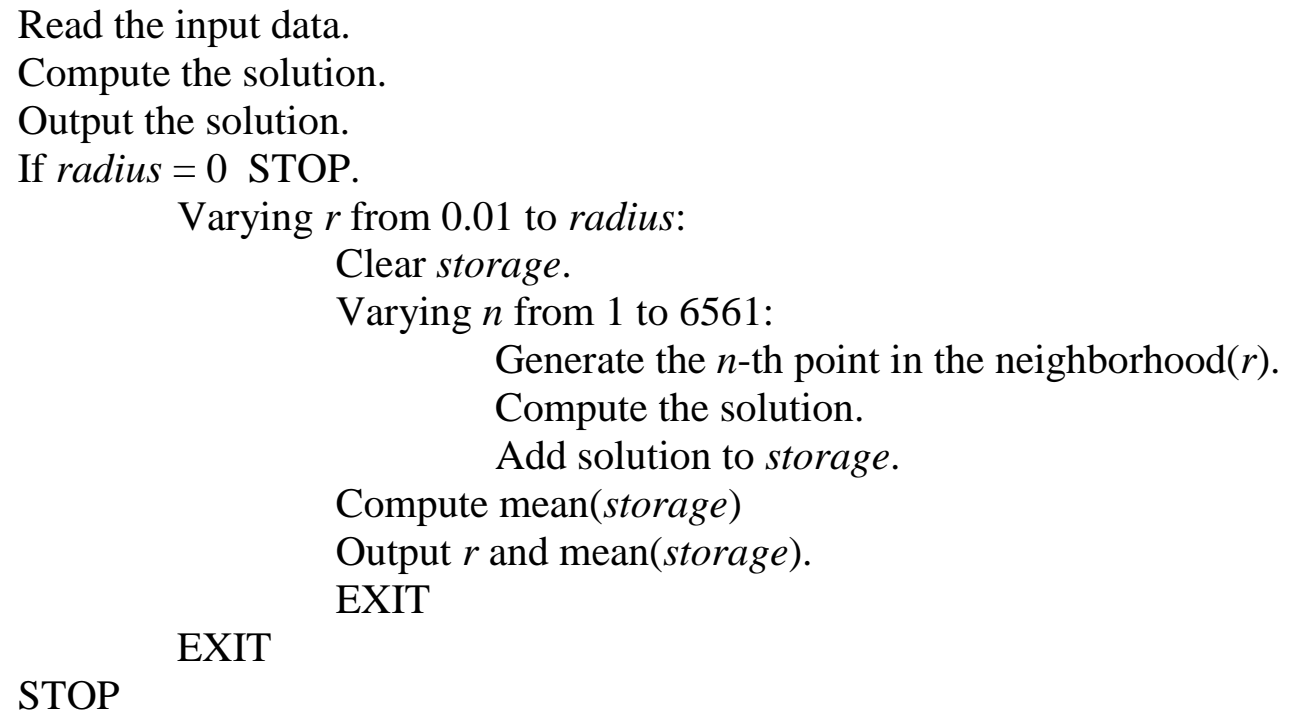

Figure 1: Algorithm pseudo code 


\section{A case study: Italy - Costa Rica (World Cup, 2014)}

Arena Pernambuco, Recife, June 20th, 2014: it is 11 o'clock in the morning. In two hours, the Chilean referee Enrique Osses will blow his whistle for the kick-off of the match between Italy and Costa Rica for the second round of Group D in the World Cup in Brazil 2014. Coaches Cesare Prandelli (Italy) and Jorge Luis Pinto (Costa Rica) must put aside all reservations and reveal their formations and initial tactics to their players. Both teams won on the first day of the tournament and now the time has come to choose whether to play offensively in an attempt to qualify immediately for the next round, or draw up a formation with more defensive tactics, thus reducing the risk of a defeat.

\subsection{The probabilities}

The odds of the leading bookmakers, retrieved from InfoBetting (2014), vary from 1.50 to 1.60 for a win for Italy, from 3.60 to 4.25 for a draw and from 6.00 to 7.08 for a win for Costa Rica. The arithmetic mean, median and Coherent Majority Average (Gambarelli, 2008), shown in Table 3, give results that are very close to each other. The rounded probabilities deriving from the arithmetic mean (i.e. the reciprocals of those numbers, normalized in order to add up to 1) are: $61 \%$ for a win for Italy, $24 \%$ for a draw and $15 \%$ for a win for Costa Rica. Those calculations lead to the same tern $(61 \%, 24 \%, 15 \%)$ even in the case of the median and of the Coherent Majority Average.

\begin{tabular}{|c|c|c|c|}
\hline & Italy wins & Draw & Costa Rica wins \\
\hline Min & 1.50 & 3.60 & 6.00 \\
\hline Arithmetic mean & $\mathbf{1 . 5 4}$ & $\mathbf{3 . 9 6}$ & $\mathbf{6 . 3 7}$ \\
\hline Median & $\mathbf{1 . 5 5}$ & $\mathbf{4 . 0 0}$ & $\mathbf{6 . 5 0}$ \\
\hline C.M.A. & $\mathbf{1 . 5 5}$ & $\mathbf{4 . 0 0}$ & $\mathbf{6 . 3 0}$ \\
\hline Max & 1.60 & 4.25 & 7.08 \\
\hline
\end{tabular}

Table 3: Synthesis of the bookmakers' odds retrieved from InfoBetting (2014).

In Table 4 we express the probabilities of a win by Italy, of a draw and of a win by Costa Rica using ordered terns, depending on the decisions taken by the coaches. We start from the hypothesis that the above-mentioned computed probabilities are a central value compared to the various offensive/defensive approaches. The tern corresponding to "Defensive-Defensive" $(0.56,0.34,0.10)$ is obtained by increasing the probability of a draw by $10 \%$ and by lowering the probability of a win for each team by 5\%; vice versa for the tern corresponding to "Offensive-Offensive" $(0.66,0.14,0.20)$. For the other two cases, consideration was given to statistics based on the results of the two teams over the last year. For the case of Italy Offensive and Costa Rica Defensive, we noticed that in the matches in which the Costa Ricans were locked in defense, the result was very rarely a draw: more often than not, Luis Pinto's team managed to catch the other team unaware and win the match. On the contrary, when Italy attacked a team barricaded in defense, it always had difficulty in imposing its game and often left itself vulnerable when the opponent counter-attacked. For these reasons, the percentages were revised as follows: +0.05 for a win for Italy, -0.15 for a draw and +0.10 for a win for Costa Rica. In 
this way, the tern $(0.66,0.09,0.25)$ was obtained. Finally, in the case of Italy Defensive and Costa Rica Offensive, no previous cases were found in which the Central Americans had attacked a more prestigious team. On the contrary, it seems that the traditional Italian game consisting of defense and counter-attack, has often rewarded Prandellli's formation. For these reasons, +0.15 was assigned for a win for Italy, -0.05 for a draw and -0.10 for a win for Costa Rica. In this way, the tern $(0.76,0.19,0.05)$ was reached.

\begin{tabular}{|l|c|c|}
\cline { 2 - 3 } \multicolumn{1}{c|}{} & Costa Rica Defensive & Costa Rica Offensive \\
\hline Italy Defensive & $(0.56,0.34,0.10)$ & $(0.76,0.19,0.05)$ \\
\hline Italy Offensive & $(0.66,0.09,0.25)$ & $(0.66,0.14,0.20)$ \\
\hline
\end{tabular}

Table 4: Probabilities of a win, a draw and a loss, depending on the strategies

It is interesting to compare the probabilities in Table 4 with the assumptions formulated in Haugen (2008). A first assumption states that if two teams play a match and both choose defensive strategies, this choice has the highest draw probability; this is indeed the case in Table 4. According to Haugen's second assumption, a larger draw-probability should be expected if one of the teams opts for a defensive strategy as opposed to the case where both play offensively. This assumption is not in line with the probabilities in Table 4. However, the Haugen writes that his assumption is controversial, since in most practical situations where one team chooses to play defensively and the other offensively, large quality differences between teams are typical and in which case, the draw probability may be very low. Considering the FIFA ranking of both countries at the time (Italy 9th, Costa Rica 27th), the low draw probability for the case where Italy attacks and Costa Rica defends is reasonable. Nevertheless, a different way to derive probabilities for each of the four offensive/defensive scenarios may be more suitable for other matches. On the other hand, taking into account a neighborhood of discrete points around these probabilities as described in Section 4 will accommodate this uncertainty at least to some extent.

\subsection{The payoffs}

Let us calculate the expected payoffs (i.e. points) in the case of a defensive formation for both teams, taking into account that a win results in 3 points, and 1 point is awarded for a draw. The expected payoff for Italy is: $3 \times 0.56+1 \times 0.34=2.02$. The expected payoff for Costa Rica is: $1 \times 0.34+3 \times 0.10=0.64$. In Table 5 , we show all expected payoffs; the first number in each pair is the expected payoff for Italy, the second for Costa Rica.

\begin{tabular}{|l|c|c|}
\cline { 2 - 3 } \multicolumn{1}{c|}{} & Costa Rica Defensive & Costa Rica Offensive \\
\hline Italy Defensive & $(2.02,0.64)$ & $(2.47,0.34)$ \\
\hline Italy Offensive & $(2.07,0.84)$ & $(2.12,0.74)$ \\
\hline
\end{tabular}

Table 5: The expected payoffs for (Italy, Costa Rica) 


\subsection{The strategies}

We now consider the problem from the point of view of Italy (see Table 5, with the Italian options shown in the rows). If Italy chooses a defensive approach, it can obtain 2.02 (if Costa Rica also chooses defense) or 2.47 (if Costa Rica chooses an offensive approach) points; if, on the other hand, Italy chooses an offensive approach, it can obtain respectively 2.07 or 2.12 points. Therefore, the Italian team does not have a dominant choice in any of the cases.

Let us consider things from the point of view of Costa Rica (see Table 5, with Costa Rica's options shown in the columns). For this team, the defensive approach is certainly preferable because it leads to an expected payoff which is no less than with an offensive approach, whatever Italy chooses to do. In fact, $0.64 \geq 0.34$ if Italy chooses a defensive approach, and 0.84 $\geq 0.74$ if Italy chooses to attack.

Therefore, a defensive approach is preferable for Costa Rica (i.e. its defensive strategy dominates its offensive one). Returning to Italy and bearing in mind the above, the corresponding choice is offensive, which leads to an expected payoff of 2.07 instead of 2.02 points. Therefore, the solution for the game is defensive for Costa Rica and offensive for Italy.

\subsection{Robustness of the results}

The results of our algorithm applied to the Italy - Costa Rica case (with radius $=0.10$ ) are shown in Table 6 . The last column shows the computation times (in seconds) that were necessary to reach the results relative to each setting for $\mathrm{r}$ using a PC i7-8650U at $1.9 \mathrm{GHz}$ with $16 \mathrm{~GB}$ RAM, running on a Windows 10 operating system.

\begin{tabular}{|l|c|c|c|c|c|c||c|}
\hline \multirow{2}{*}{$r$} & \multicolumn{3}{|c|}{ ITALY } & \multicolumn{3}{c||}{ COSTA RICA } & $\begin{array}{c}\text { Oomput. } \\
\text { time }\end{array}$ \\
\cline { 2 - 7 } & $\% \mathrm{O}$ & $\% \mathrm{D}$ & $\mathrm{E}$ (points) & $\%$ O & $\% \mathrm{D}$ & $\mathrm{E}$ (points) & \\
\hline \hline .00 & 100 & 0 & 2.07 & 0 & 100 & 0.84 & 0.0 \\
\hline \hline .01 & 98 & 2 & 2.07 & 0 & 100 & 0.83 & 0.2 \\
\hline .02 & 82 & 18 & 2.07 & 0 & 100 & 0.79 & 0.4 \\
\hline .03 & 67 & 33 & 2.08 & 0 & 100 & 0.75 & 0.6 \\
\hline .04 & 63 & 37 & 2.09 & 0 & 100 & 0.74 & 0.9 \\
\hline .05 & 55 & 45 & 2.09 & 0 & 100 & 0.71 & 1.1 \\
\hline .06 & 48 & 52 & 2.10 & 1 & 99 & 0.69 & 1.3 \\
\hline .07 & 48 & 52 & 2.11 & 4 & 96 & 0.69 & 1.5 \\
\hline .08 & 48 & 52 & 2.13 & 10 & 90 & 0.68 & 1.7 \\
\hline .09 & 48 & 52 & 2.14 & 10 & 90 & 0.68 & 2.0 \\
\hline .10 & 47 & 53 & 2.15 & 16 & 94 & 0.67 & 2.2 \\
\hline
\end{tabular}

Table 6: Results of the computation for Italy - Costa Rica 
As can be seen, the best strategies for the two teams given the estimates in Table 4 (i.e. on the first line of the table, with $r=0.00$ ) are those obtained in section 5.3, that is an offensive strategy for Italy and defensive play for Costa Rica. As the radius increases, in other words as the uncertainty regarding the initial data grows, the best strategy for Italy moves slightly from attack to defense, while that of Costa Rica remains set for a long time on defense, before starting to move towards attack. As the uncertainty grows, the corresponding expected payments increase for Italy and decrease for the opposing team.

\subsection{Handling in-game events}

Contrary to our results in section 5.3 and 5.4, the coaches of both teams opted for defensive initial formations and tactics. The teams entered the field and played very cautiously, to the extent that in the first half an hour the only noteworthy action was a header following a corner kick by Costa Rica, which landed two meters outside the goal mouth. The situation remained more or less unchanged until the 44th minute of the game, when Bryan Ruiz Gonzàlez scored for Costa Rica. To estimate the impact on the win probabilities of this goal (as well as the fact that only one half remained for the Italian team to rectify the situation), we rely on a model by Beuoy (2014) based on local ordered logistic regression, taking into account about 30,000 matches. This model takes as an input pre-match odds, the game time, and the goal differential, and outputs in-game win, draw and loss probabilities. Other than perhaps through the pre-match odds, it does not take into account home advantage, which makes it highly suitable for World Cup matches (provided that they don't include the host country). The resulting (rounded) probabilities reflecting the situation after 44 minutes with Costa Rica leading 1-0 are 27\% for a win for Italy, $33 \%$ for a draw and $40 \%$ for a win for Costa Rica. After adjusting these probabilities for each combination of offensive/defensive approaches in a similar way ${ }^{1}$ as described in Section 5.1, we obtain the updated expected payoffs in Table 7.

\begin{tabular}{|c|c|c|}
\hline & Costa Rica Defensive & Costa Rica Offensive \\
\hline Italy Defensive & $(0.94,1.78)$ & $(1.39,1.18)$ \\
\hline Italy Offensive & $(1.19,1.43)$ & $(1.34,1.28)$ \\
\hline
\end{tabular}

Table 7: The expected payoffs for (Italy, Costa Rica), for Costa Rica leading 0-1 after 44 minutes.

Clearly, defending is a dominant strategy for Costa Rica: no matter what the Italians do, the expected payoffs are always higher if Costa Rica assumes a defensive stance. Italy does not

1 If both teams adopt a defensive strategy, we add $10 \%$ to the status quo (i.e. Costa Rica wins) while reducing each of the other outcomes with $5 \%$. If both teams opt to attack, we reduce the status quo with $10 \%$, to be added evenly over the other options. If Italy adopts an offensive strategy, and Costa Rica defends, the probability of a draw increases with $5 \%$ at the expense of a win by Costa Rica. If Costa Rica attacks while Italy defends, we increase the win probability of Italy with $5 \%$, and the probability of a draw with $10 \%$, at the expense of Costa Rica winning the game. 
have a dominant strategy, but given that Costa Rica has a clear interest to defend, they better opt to attack. Hence, the Costa Rican goal does not change the initial strategic advice given by our method (see section 5.3). Table 8 gives the computational results, as we include more uncertainty into our win probabilities. Compared to Table 6, it points much more unambiguously towards an attacking strategy for Italy.

\begin{tabular}{|l||c|c|c||c|c|c||c||}
\hline \multirow{2}{*}{$r$} & \multicolumn{3}{|c|}{ ITALY } & \multicolumn{3}{c||}{ COSTA RICA } & $\begin{array}{c}\text { Oomput. } \\
\text { time }\end{array}$ \\
\cline { 2 - 7 } & $\% \mathrm{O}$ & $\% \mathrm{D}$ & $\mathrm{E}$ (points) & $\%$ O & $\% \mathrm{D}$ & $\mathrm{E}$ (points) & \\
\hline .00 & 100 & 0 & 1.19 & 0 & 100 & 1.43 & 0.0 \\
\hline .01 & 100 & 0 & 1.19 & 0 & 100 & 1.43 & 0.2 \\
\hline .02 & 100 & 0 & 1.19 & 0 & 100 & 1.43 & 0.4 \\
\hline .03 & 100 & 0 & 1.19 & 0 & 100 & 1.43 & 0.6 \\
\hline .04 & 100 & 0 & 1.19 & 0 & 100 & 1.43 & 0.8 \\
\hline .05 & 100 & 0 & 1.19 & 2 & 98 & 1.43 & 0.9 \\
\hline .06 & 96 & 4 & 1.19 & 2 & 98 & 1.43 & 1.2 \\
\hline .07 & 89 & 11 & 1.19 & 4 & 96 & 1.45 & 1.3 \\
\hline .08 & 89 & 11 & 1.19 & 6 & 94 & 1.45 & 1.5 \\
\hline .09 & 81 & 19 & 1.20 & 10 & 90 & 1.46 & 1.7 \\
\hline .10 & 80 & 20 & 1.20 & 10 & 90 & 1.46 & 1.9 \\
\hline
\end{tabular}

Table 8: Results of the computation for Italy - Costa Rica, for Costa Rica leading 0-1 after 44 minutes.

In the second half of the game, the Italian coach Prandelli indeed introduced some changes to modify the formation of the Italian team, making it more offensive. These changes, however, turned out too little too late to alter the result: the match ended 1-0 in favor of the Central American team. Eventually, Costa Rica qualified for the next round (and even reached the quarter finals), while Italy did not survive the group stage.

\subsection{The impact of the point system}

Our approach can also be used to assess the impact of various point system on the willingness of teams to attack. We illustrate this again with the World Cup game between Italy and Costa Rica. Figure 2 presents the willingness of Italy (left) and Costa Rica (right) to opt for attacking play at the start of the game. We compare the current rule ( 3 points for a win, 1 point for a draw), with the previous rule, which awarded 2 points for a win, and a rule that awards 4 points for a win. We also included a rule that only rewards a win ( 0 points for a draw or a loss). For the case with $r=0.00$, we see that the FIFA rule change awarding 3 points for a win instead of 2 has had a huge impact on the strategy of Italy: they move from $0 \%$ attacking play under the old rule to $100 \%$ attacking play under the new rule. For Costa Rica, none of the rules can persuade them to abandon their defensive stance. If we include more uncertainty on our parameter estimates (i.e. 
larger values for $r$ ), we see that the difference between both rules is less pronounced for Italy, and that it remains negligible for Costa Rica.
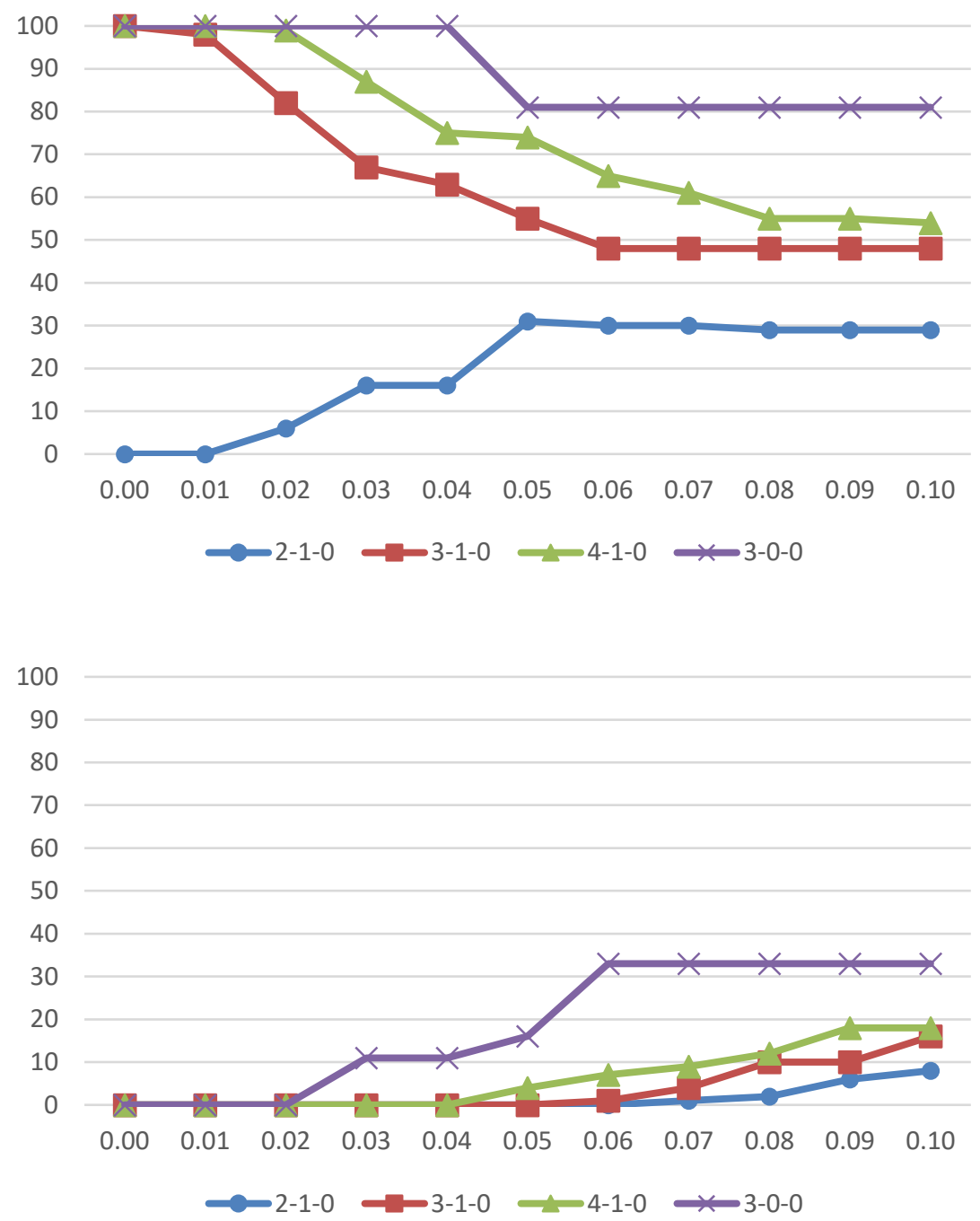

Figure 2: \%O for Italy (left) and Costa Rica (right) for various point systems (win - draw - loss), for $r$ ranging from 0.00 to 0.10 on the $\mathrm{x}$-axis.

Figure 2 also show that in this case, rules that further favor a win compared to a draw will indeed result in more attacking play. However, even in the most extreme case, where only a win counts, there will be situations where a team's best strategy is to defend.

\section{Concluding remarks}

The method presented here provides insight in the choice of tactics to be adopted at the start of the match, and can be used as a tool to support coaches. As the input of our algorithm is based 
on a subjective evaluation of the available data (e.g. bookmaker's odds), it is subject to uncertainty. To some extent, our algorithm can deal with this uncertainty, by computing the optimal strategies for a wide range of probabilities around the original estimates. On top of that, during the match, the situation on the pitch (e.g. a goal scored) may require appropriate changes to be made. At this point, our method should be rerun based on updated win probabilities, which take into account the most recent match events. As our computer program runs very quickly, our method can be used during an ongoing game to give real-time strategic advice.

Although the model has its limitations (as is the case for any theoretical model), we believe that game theory provides a highly suitable framework for analyzing this type of strategic considerations between two opponents in sports. Furthermore, while in this text the focus is on soccer, our approach can be applied to any sport with a win(-draw)-loss structure and a scoring rule, where two opponents faces a choice between two (or more) strategies.

Our method also allows to obtain some insight in the effect of various point systems on the strategic considerations for a particular match. Obviously, we are not making any claims in favor or against the switch to the 3-1-0 rule based on a single case study. However, our results show that even in settings where anything other than a win does not yield any points, for some teams a defensive stance is still the best way to achieve a win. In this sense, no point system will be able to guarantee offensive play.

Finally, while our case study hints the possibilities of our game-theoretical approach, a largescale empirical study of the success of coaches following this approach is left for future research. This is mostly due to the lacking of a straightforward tool to quantify a team's offensive or defensive play, which would in our opinion require a thorough analysis of the formation, the players, and their behavior on the pitch. Given a way to quantify offensive/defensive play, one could evaluate the success of coaches that follow our strategic advice, and obtain a better consideration of the merits of our approach.

\section{References}

Aylott, M. and H. Aylott (2007). "Meeting of Social Science and Football: Measuring the Effects of Three Points for a Win". Sport in Society: Cultures, Commerce, Media, Politics, Volume 10, Issue 2, pp. 205-222.

Auer, B.R. and T. Hiller (2015). "On the evaluation of soccer players: a comparison of a new gametheoretical approach to classic performance measures". Applied Economics Letters, Volume 22, Issue 14, pp. 1100-1107.

Brams, S. and M.S. Ismail (2018). "Making the rules of sports fairer". SIAM Review, Volume 60, Issue 1, pp. 181-202.

Brocas, I., and J.D. Carrillo (2004). "Do the three-point victory and golden goal rules make soccer more exciting?’. Journal of Sports Economics, Volume 5, pp. 169-185.

Beuoy, M. (2014). "In-Match Soccer Probability". Inpredictable. http://stats.inpredictable.com/soccer/wpCalc.php. Consulted on October $15^{\text {th }} 2018$.

Chiappori, P., Levitt, S., Groseclose, T. (2002). Testing mixed-strategy equilibria when players are heterogeneous: The case of penalty kicks in soccer. American Economic Review, 92, 1138-1151. 
Coloma, G. (2007). Penalty kicks in soccer: An alternative methodology for testing mixed-strategy equilibria. Journal of Sports Economics, 8, 530-545.

Deck, A., C. Deck, and Z. Zhen (2014). "Decision Making in a Sequential Game: The Case of Pitting in NASCAR”. Journal of Sports Economics, Volume 15, Issue 2, pp. 132-149.

Dewenter, R. and J. Emami Namini (2013). "How to Make Soccer More Attractive? Rewards for a Victory, the Teams' Offensiveness, and the Home Bias". Journal of Sports Economics, Volume 14 , Issue 1 , pp. 65-86.

Dilger, A. and H. Geyer (2009). "Are Three Points for a Win Really Better Than Two? A Comparison of German Soccer League and Cup Games". Journal of Sports Economics, Volume 10, Issue 3, pp. 305-318.

Dohmen, T. and H. Sonnabend (2018). "Further Field Evidence for Minimax Play" Journal of Sports Economics, article first published online: July 21, 2016. DOI: https://doi.org/10.1177/1527002516656729.

Fernandez-Cantelli, E. and G. Meeden (2002). "An improved award system for soccer". Chance, Volume 16, pp. 23-29.

Gambarelli, G. (2008). “The 'Coherent Majority Average' for Juries' Evaluation Processes”. Journal of Sport Sciences, Volume 26, Issue 10 (August), 1091-1095

Gambarelli, G. and G. Owen (2004). "The coming of Game Theory". Essays on Cooperative Games - in honor of Guillermo Owen (G. Gambarelli, ed.), Special Issue of Theory and Decision, Vol. 36, Dordrecht: Kluwer Academic Publishers, 1-18.

Haugen, K.K. (2008). "Point Score Systems and Competitive Imbalance in Professional Soccer". Journal of Sports Economics, Volume 9, Issue 2, pp. 191-210.

Hernández-Lamoneda, L. and F. Sánchez-Sánchez (2010). "Rankings and values for team games". International Journal of Game Theory, Volume 39, pp. 319-350.

Hiller, T. (2015) "The importance of players in teams of the German Bundesliga in the season 2012/2013 - A cooperative game theory approach". Applied Economics Letters, Volume 22, pp. 324-329.

Hirotsu, N. and M. Wright (2006). "Modeling Tactical Changes of Formation in Association Football as a Zero-Sum Game". Journal of the Quantitative Analysis in Sports, Volume 2, Issue 2.

Hirotsu, N., M. Ito, C. Miyaji, and K. Hamano (2009) "Modeling Tactical Changes in Association Football as a Non-Zero-Sum Game". Journal of Quantitative Analysis in Sports, Volume 5, Issue 3.

InfoBetting (2014). Bookmakers' odds for Italy-Costa Rica (World Cup, 2014), retrieved from http://www.infobetting.com/calcio/quote/international/mondiali/italia-costa-rica/1x2/dett-t-45-1q-237-637-416849-1x2.htm.

Jordan, J.D., S.H. Melouk and M.B. Perry (2009). “Optimizing Football Game Play Calling”. Journal of Quantitative Analysis in Sports, Volume 5, Issue 2.

Nash, J. F. (1950). “The Bargaining Problem”. Econometrica, 18, 155-162. 
Owen, G. (1968). Game Theory. Academic Press (1st ed., New York) (II ed. 1982, New York) (III ed. owe, San Diego) (IV ed. 2013. Emerald - United Kingdom).

Palacios-Huerta, I. (2003). Professionals play minimax. Review of Economic Studies, 70, 395415.

Palacios-Huerta, I. (2014). Beautiful Game Theory: How Soccer Can Help Economics. Princeton University Press. 2014.

von Neumann, J. (1928). "Zur theorie der Gesellschaftsspiele". Mathematische Annalen, 100, 295-320. Translated by S. Bargmann in (1959) in R.D. Luce and A.W. Tucker (Eds.), as "On the Theory of Games of Strategy".

Walker, M., Wooders, J. (2001). Minimax play at Wimbledon. American Economic Review, 91, 1521-1538. 